\title{
On the Nature of the Emission of the Crab Nebula
}

\author{
I. S. SHKLovsky \\ Sternberg Astronomical Institute, Moscow, U.S.S.R.
}

$\mathrm{T}$ HE emission of the Crab Nebula is perhaps connected with the internal motions of the gas. As shown by the observations, the power of the emission sources differs by some hundred times in different points of the amorphous mass ("wisps" and other details). Oort and Walraven believe the "wisps" to be condensations of relativistic electrons ejected from the central star. However, there are some other variable details elsewhere in the nebula (as described by Lampland, and Oort and Walraven).

Oort and Walraven's hypothesis met some important difficulties: (a) the direction of the magnetic lines in this region of the nebula is perpendicular to the wisp's velocity; (b) the dimension of the wisp is about three light months, while it appears during a month and faster, so that the relativistic electrons moving along the lines of force have no time to spread along the wisp; and (c) if the strength is not changing, the energy of the relativistic electrons in the wisp must be some hundred times greater than the density of the magnetic energy, consequently the field strength must grow in the wisp to keep the electrons in the volume.

Such an increase of the field leads to an increase of the emission, without ejection of relativistic electrons from the star. (a) We have $\epsilon_{\nu} \propto K H_{\perp}{ }^{(\gamma+1) / 2}$, where $\gamma_{\text {opt }}$ is about 4.5-5 (according to Sheglov); (b) evidently, $K \propto H$; (c) as $P_{1}^{2} / H=$ const, the energy of electrons will increase with the growth of the field strength. A twofold or threefold increase of the field strength leads to a hundred-fold increase of $\epsilon_{\nu}$. Consequently, the ejection hypothesis is not necessary.

When the field obtains its initial value, the energy and concentration of the relativistic electrons will decrease and $\epsilon_{\nu}$ becomes small. During the existence of the wisp the radiation loss of energy is small. Thus, the intensity variation may be explained by the local fluctuations of the magnetic field. The difficulty of conservation of the "optical" electrons of high energy disappears. The relativistic electron becomes "optical" when fluctuations of the magnetic field are taking place around it. As soon as the fluctuations disappear, the energy of the electrons diminishes according to $P_{\perp}^{2} \propto H$ and the electrons cease to be "optical." But other electrons become "optical" elsewhere.

The presence of fluctuations in a field may be perhaps explained by the connection of the field with the gaseous filament. The field has a tendency to leave the nebula in the regions where the density of the gas is less. At a later time the field will find some other weak region and this process leads to a continuous reconstruction of the magnetic field. Under such conditions magnetohydrodynamic waves can originate, the velocity of which could be very large and near to the observed velocities. The concentration of relativistic electrons in these fluctuations may be about $10^{-2}-10^{-3}$, if we take into account the recent observations of Weels at $26.75 \mathrm{mc} / \mathrm{sec}$. It is not excluded that the mean part of the amorphous mass consists of relativistic particles.

\section{DISCUSSION}

S. B. PICKelNer, Crimean Astrophysical Observatory, Simeis, U.S.S.R.: It should be noted that the filaments occur in regions where the magnetic field concentrates.

G. MÜNCH, California Institute of Technology, Pasadena, California: I think the problem of the Crab Nebula is far too complicated to discuss in a very few minutes, but I have a general question of whether we can attach much confidence to the considerations of high-energy particles-pressure of high-energy particles, etc. The point is that we do not know anything at all about the energy content of the fast protons.
S. B. PiCKelneR: Shklovsky assumes that the energy of the protons is about the same as that of the electrons.

G. MÜNCH : In any mechanism of acceleration of particles, the electrons will not build up as high energies because of bremsstrahlung and collisional losses.

S. B. PICKELNER : But the energy of protons cannot be greater than the energy of electrons, because the energy of the relativistic electrons is very large in this nebula. 